Nouvelles perspectives en sciences sociales

\title{
À propos de
}

Quantum Mind and Social Science. Unifying Physical and

Social Ontology. Alexander Wendt, Cambridge University

Press, 2015, 354 pages

\section{Cyrille Rigolot et Leonardo Orlando}

Volume 15, numéro 1, novembre 2019

URI : https://id.erudit.org/iderudit/1068184ar

DOI : https://doi.org/10.7202/1068184ar

Aller au sommaire du numéro

\section{Éditeur(s)}

Prise de parole

\section{ISSN}

1712-8307 (imprimé)

1918-7475 (numérique)

Découvrir la revue

Citer ce compte rendu

Rigolot, C. \& Orlando, L. (2019). Compte rendu de [À propos de / Quantum Mind and Social Science. Unifying Physical and Social Ontology. Alexander Wendt, Cambridge University Press, 2015, 354 pages]. Nouvelles perspectives en sciences sociales, 15(1), 249-260. https://doi.org/10.7202/1068184ar d'utilisation que vous pouvez consulter en ligne. 


\section{À propos de}

\section{Quantum Mind and Social Science. Unifying Physical and Social Ontology.}

Alexander Wendt, Cambridge University Press, 2015, 354 pages.

Cyrille Rigolot

Inra Clermont-Ferrand, UMR Territoires

LEONARDO ORLANDO

Science Po, Paris

$A_{\text {tionales dans les années } 1990 \text {, Alexander Wendt, qui est }}^{\text {prèr }}$ considéré à la fois par les universitaires et les praticiens comme le chercheur le plus important dans ce domaine, a rompu un silence éditorial de seize ans, pour revenir, après son ouvrage fondamental Social Theory of International Politics ${ }^{1}$, avec une nouvelle proposition révolutionnaire pour les sciences sociales. Or, cette fois-ci, la démarche semble tout aussi radicale qu'inattendue : lier sciences sociales et mécanique quantique. Dans Quantum Mind and Social Science. Unifying Physical and Social Ontology (QMASS), Wendt défend une proposition simple : la conscience serait un phénomène quantique macroscopique.

Alexander Wendt, Social theory of international politics, Cambridge University Press, 1999. 
Bien que cette proposition ait déjà été avancée ailleurs ${ }^{2}$, Wendt se distingue ici par l'ambition de proposer un traitement rigoureux, qui puisse être soumis à une critique académique sérieuse. Pour cela, l'auteur a plongé dans l'étude approfondie de la physique quantique, pendant plus de dix ans, temps que l'écriture de l'ouvrage lui a demandé. QMASS est adressé à toutes les sciences sociales : comme l'indique le sous-titre, l'auteur propose une nouvelle ontologie physique et sociale unifiée. Pour justifier son entreprise, dans la préface, Wendt invoque le principe du "causal closure of physics", selon lequel toutes les sciences sociales seraient contraintes au moins implicitement par les lois de la physique. Ceci ne signifie évidemment pas qu'elles soient réductibles à la physique, mais qu'elles ne peuvent en aucun cas violer explicitement ses lois (comme la gravité...), sous peine de discrédit. Or, note Wendt, une partie des sciences sociales viole en fait implicitement les lois de la physique classique en routine, en invoquant des phénomènes intentionnels ou des structures sociales (comme l'État), dont Wendt montre qu'elles n'ont tout simplement pas d'existence concevable selon la physique classique. Ceci s'explique du fait de l'aporie dans laquelle se retrouve la science actuelle par rapport au "mindbody problem ", soit l'impossibilité à expliquer l'esprit à partir d'une métaphysique matérialiste comme celle sur laquelle reposent toutes les sciences (dont les sciences sociales). Pour l'auteur, la clé de voûte pour dépasser le problème corps-esprit se retrouve dans la théorie quantique. Afin de démontrer cela, il a construit son livre comme un édifice solide où les éléments correspondent chacun à un domaine de recherche (philosophie de l'esprit, neurosciences, théorie des jeux), qui s'éclairent les uns les autres. Par-delà la résolution du problème corps-esprit, cela aurait notamment deux intérêts : i) contribuer à légitimer

$\overline{2}$ Stuart Hameroff et Roger Penrose, «Orchestrated Reduction of Quantum Coherence in Brain Microtubules: A Model for Consciousness ", Mathematics and Computers in Simulation, vol. 40, $\mathrm{n}^{\text {os }} 3$ et 4, p. 453-480, 1996 ; Henry Stapp, Mind, Matter, and Quantum Mechanics, Springer, Berlin et Heidelberg, Springer, 2004. 
des sciences sociales restées en marge de l'orthodoxie (comme le pragmatisme) ; ii) suggérer de nouvelles pistes de recherche.

Dans ce compte-rendu, notre premier objectif est de proposer un aperçu général de l'ouvrage en langue française (il n'a pas été traduit), à destination des communautés de recherche transdisciplinaire. L'ouvrage est composé de cinq parties, correspondant à la présentation de la théorie de la conscience quantique (parties $1,2,3)$, puis au développement de certaines implications pour les sciences sociales (parties 4 et 5). Dans un deuxième temps, nous faisons état des intérêts et limites de l'ouvrage identifiés lors de sa réception critique, et de certaines pistes de recherche qui ont émergé suite à sa parution.

\section{La théorie de la conscience quantique (parties 1, 2, 3)}

La première partie de l'ouvrage constitue une présentation relativement accessible de la physique quantique. En s'appuyant sur trois expériences fondatrices (dont la célèbre double-fente de Young), Wendt introduit les résultats les plus marquants et les concepts essentiels. En particulier, la notion de fonction d'onde peut être appréhendée comme un champ de potentialités en "superposition ", qui s'effondre au moment de l'observation (phénomène de "réduction du paquet d'onde»). Selon le principe de non-localité, des entités peuvent être instantanément corrélées à distance, en vertu de leur "entanglement " (intrication quantique). Wendt rappelle que la non-localité peut aussi être temporelle : dans le monde quantique, le choix d'une procédure de mesure peut affecter des événements dans le passé. La physique quantique remet en cause les grands principes de la physique classique (matérialisme, déterminisme, distinction sujet-objet...). De nombreuses interprétations existent (Copenhague, De BroglieBohm, mondes multiples...) dont aucune ne fait consensus.

Selon la théorie la plus communément admise (Copenhague), les phénomènes quantiques ne peuvent pas se produire au-delà de l'échelle moléculaire, a fortiori dans un environnement aussi " chaud, humide et bruyant " que le cerveau, pour lequel il est généralement considéré que les lois de la physique classique 
s'appliquent. Ceci s'explique par le phénomène dit de "décohérence", lié aux interactions des particules avec leur environnement. Prenant le contre-pied de cette théorie, la deuxième partie de l'ouvrage vise à montrer la plausibilité de la "théorie du cerveau quantique ", en faisant état des principales hypothèses et controverses. Wendt parvient à convaincre de cette plausibilité, mais sa démonstration amène à considérer deux autres théories controversées : i) le panpsychisme, selon lequel la matière est dotée d' "esprit » au niveau le plus fondamental (minded matter); ii) le vitalisme, selon laquelle le vivant est constitué d'une force inobservable et non matérielle (l'élan vital). En dépit d'un discrédit scientifique pour ces deux théories au $\mathrm{XX}^{\mathrm{e}}$ siècle, l'auteur montre habilement qu'elles sont en fait au moins plausibles scientifiquement. Il s'appuie pour ceci notamment sur une interprétation particulière de la physique quantique (interprétation de Bohm). Par ailleurs, Wendt fait état de récentes découvertes dans le domaine de la biologie quantique, démontrant rigoureusement l'occurrence et le rôle essentiel d'effets quantiques dans des phénomènes biologiques fondamentaux tels que la photosynthèse, la catalyse enzymatique, ou encore la magnétoréception dans la navigation migratoire de certains oiseaux. Si la cohérence quantique permet d'expliquer en partie le comportement des oiseaux, questionne Wendt, pourquoi ne pourrait-elle pas s'appliquer aussi aux humains?

Après l'explication biologique, Wendt aborde dans la troisième partie le domaine de la décision, où certains résultats tendent à valider empiriquement, selon lui, l'hypothèse de la conscience quantique. Ainsi, "la théorie de la décision quantique " permet d'expliquer de façon très satisfaisante des anomalies de longue date (" effets d'ordre », inversion de préférences...), restées difficilement concevables à partir des théories dominantes de l'utilité attendue et de l'action rationnelle. Un autre domaine prometteur est la version quantique de la théorie des jeux, dont un résultat notable est de donner beaucoup plus de place à la coopération entre individus que la version classique. En arrière-plan de ces résultats, une perspective quantique donne notamment une place 
naturelle en science sociale à la volonté et au libre arbitre, que Wendt associe au phénomène de réduction du paquet d'onde (tandis que ces notions apparaissent comme "mystérieuses ou éliminables " dans une vision classique). Selon la conception quantique, volonté et libre arbitre sont intimement liés à la question du temps et de la projection mentale dans le futur. Wendt introduit ainsi la notion de "temporalité non locale", soit la superposition des états à des moments temporels différents (alors que, selon la temporalité « locale» du paradigme classique, les évènements n'ont pas de connexion intrinsèque entre eux, mais ce sont des points séparés dans une séquence temporelle). Cette approche apporte notamment un éclairage nouveau sur les expériences déconcertantes de Benjamin Libet, qui avaient démontré que la prise de conscience dans la « décision » d'effectuer une action motrice intervient en fait après que l'action ait été initiée (ce que beaucoup considèrent comme une preuve que le libre arbitre est une illusion, suivant une interprétation classique). Ces considérations temporelles amènent Wendt à développer une autre idée étonnante : selon lui, il serait possible de changer le passé dans un sens littéral (ontologique), dans sa composante intentionnelle ou relationnelle. Pour ceci, Wendt élabore soigneusement différentes modalités possibles (effets d'addition et effets de remplacement), qu'il illustre par des exemples collectifs (l'Histoire) et individuels. Par exemple, l'assertion « la guerre de 30 ans commence en 1618 » montre comment une bataille localisée en 1618 peut devenir une partie d'un évènement beaucoup plus large après 1648. Au niveau individuel, la conception de Wendt consolide l'idée selon laquelle c'est à travers la répétition des comportements que des actions passées deviennent constitutives d'un trait de caractère ou un autre, comme être quelqu'un de courageux ou d'imprudent. Au final, avec une conception quantique, l'homme apparait comme "physique mais pas totalement matériel, avec des états mentaux "superposés " plutôt que bien définis, [...] libre et très vivant ". 


\section{Implications générales pour les sciences sociales (parties 4 et 5)}

Dans la partie 4, Wendt aborde la question fondamentale du langage, en mobilisant la littérature de la sémantique quantique. Selon cette approche, les concepts se comporteraient de la même façon que des particules (et réciproquement). Ainsi, l' « état de base " d'un concept peut être représenté comme une superposition de "meaning" (sens) potentiels, chacun étant un vecteur d'une fonction d'onde, avec différents poids respectifs (Wendt prend l'exemple de "suit» en anglais, pouvant être à la fois un costume, une poursuite judiciaire ou une suite de poker). Par ailleurs, le partage commun d'un langage et de sens partagés pourrait impliquer des connexions non locales, par intrication quantique. Le deuxième chapitre de cette partie s'intéresse à la question des " autres esprits " ( other minds») : comment se fait-il que nous réussissions couramment à connaitre l'état d'esprit d'autres individus, étant donné l'aspect privé de la conscience ? Wendt défend l'idée d'un "individualisme ouvert ": Les individus ne sont pas totalement "séparables" au sens quantique, dans la mesure où ils partagent par moment une même superposition (tant qu'ils sont " co-présents"). Une analogie est faite avec les océans, à la fois distincts et constituant une seule entité aux frontières (océan unique).

Dans la cinquième partie, Wendt explore les implications de sa théorie pour une question majeure en sciences sociales : le problème agent-structure. Selon lui, deux débats principaux structurent ce problème : tout d'abord, l'opposition entre l'individualisme (les structures sont réductibles aux agents) et l' " émergentisme » (pour lequel des phénomènes émergent de manière nécessaire (et non aléatoire) à partir d'entités fondamentales, auxquelles ils ne peuvent être réduits). Deuxièmement, concernant les relations entre individus, Wendt distingue les approches atomistes (les individus sont ontologiquement antérieurs à la société, et fondamentalement séparés par la frontière de la peau) et holistes (les pensées d'un individu ne peuvent pas même être définies, si ce n'est en relation avec d'autres individus). Selon Wendt, le débat est toujours soumis implicitement aux contraintes de la physique 
classique (comme la causalité linéaire), expliquant en partie pourquoi l'émergentisme et le holisme sont restés plutôt à la marge de la théorie sociale, malgré quelques percées très significatives (constructivisme et institutionnalisme historique en science politique, distinction d'une théorie macro non réductible à la théorie micro en économie...). De plus, contraint par la physique classique, l'émergentisme a nécessairement été associé à une ontologie stratifiée, avec différents niveaux. Selon la théorie quantique de Wendt, au contraire, l'ontologie sociale est à la fois holiste et émergentiste, mais plate. Dans une ontologie plate, comme par exemple celle de Bruno Latour, toutes les entités, humaines et non-humaines, micro et macroscopiques, se voient investies d'une égale potentialité quant au rôle qu'elles peuvent avoir dans la structure (ou réseau) qu'elles intègrent. En physique quantique, l'émergence est liée à l'" entanglement", qui fait que le tout et les parties (structures et agents) " co-émergent " en même temps. Ainsi, les structures sociales entrent et sortent ("popping in and out») continuellement dans l'existence par les pratiques à travers lesquelles elles sont instanciées. En particulier, pour Wendt, les structures sociales sont, physiquement, des superpositions d'états mentaux partagés, c'est-à-dire des « fonctions d'onde sociales ». Les réalités deviennent effectivement telles, c'est-à-dire des potentialités actualisées, à travers la co-émergence qui résulte de la réduction du paquet d'onde. Ce phénomène se produit non pas dans les relations verticales entre niveaux, mais dans les relations horizontales globales entre agents, dont les états sont constitués par des intrications non locales. Cette perspective a beaucoup de résonnance selon Wendt avec le «tournant de la pratique ", selon lequel le problème agent-structure peut être résolu en mettant de côté les agents et les structures sociales et en s'intéressant à la place aux pratiques. D'autres résonnances sont développées par l'auteur avec la conception d'Anthony Giddens du problème agent-structure, la théorie de l'acteur-réseau (Bruno Latour), le pragmatisme (William James, John Dewey) ou encore l'anthropologie philosophique (Hans Joas). 


\section{Réception critique de l'ouvrage et pistes de recherche émergentes}

Du fait de la radicalité de ses implications et de la crédibilité de son auteur, l'ouvrage a déjà généré plusieurs commentaires critiques, portant sur la plausibilité physique ou biologique des thèses avancées $^{3}$, ou sur les intérêts et limites pour les sciences sociales ${ }^{4}$. Selon l'analyse postérieure de Wendt, ces critiques sont très contrastées, dans le sens où la majorité d'entre elles portent un avis soit positif, soit très négatif sur la globalité de l'ouvrage 5 . Dans sa réponse à ces critiques, Wendt insiste en particulier sur la pertinence de son approche vis-à-vis du "mind-body problem». Il revient notamment sur la place essentielle du panpsychisme pour dépasser ce problème, en s'appuyant sur plusieurs physiciens quantiques de renom. Aussi, le panpsychisme peut être vu à la fois le pilier et la conclusion de sa démarche (on peut noter qu'il existe déjà une tradition panpsychiste en science sociale, en particulier chez Gabriel Tarde, qui, dans Monadologie et Sociologie ${ }^{6}$, adhère explicitement à l'idée que la conscience est déjà contenue dans la matière). Pour le reste, sans entrer dans le détail, si certains commentaires pointent légitimement certaines faiblesses de l'ouvrage, ils ne remettent pas en cause radicalement la plausibilité

3 Vicky Kirby, " Originary Différance: "A quantum vitalism” ", Journal for the Theory of Social Behaviour, vol. 48, n 2, 2018, p. 162-166, 2018 ; Daniel Little, "Entangling the Social: Comments on Alexander Wendt, Quantum Mind and Social Science ", Journal for the Theory of Social Behaviour, $\mathrm{n}^{\circ} 48$, 2018, p. 167-176 ; David Waldner, "Schrödinger's Cat and the Dog That Didn't Bark: Why Quantum Mechanics Is (Probably) Irrelevant to the Social Sciences ", Critical Review, vol. 29, n 2, 2017, p. 199-233.

4 Bentley B. Allan, "Social Action in Quantum Social Science ", Millennium. Journal of International Studies, 0305829818781690, 2018 ; Stephen DeCanio, "What Is It Like to Be a Social Scientist? ", Critical Review, vol. 29, $\mathrm{n}^{\circ} 2$ 2, 2017, p. 121-140 ; Robert Jervis, "One World or Many? ", Critical Review, vol. 29, n 2, 2017, p. 170-188; Sven Steinmo, "Wendt's Challenge to Social Science: Quantum Physics, Consciousness, and Society ", Critical Review, vol. 29, n 2, 2017, p. 189-198.

5 Alexander Wendt, "The Mind-Body Problem and Social Science: Motivating a Quantum Social Theory ", Journal for the Theory of Social Behaviour, vol. 48, $\mathrm{n}^{\circ} 2$, 2018, p. 188-204.

6 Gabriel Tarde, Monadologie et sociologie. Les lois sociales, Paris, Les empêcheurs de penser en rond, 1999 [1893]. 
des arguments. À notre sens, Wendt parvient à bien discerner ce qui relève de données établies, de conjectures (non démontrées), ou de spéculations personnelles (même s'il se montre peut-être parfois trop optimiste sur la portée de certains résultats ${ }^{7}$ ). Par endroits, « la pilule a plus de mal à passer ", comme quand Wendt demande au lecteur d'adhérer coup sur coup à la théorie du cerveau quantique, au panspychisme et à une certaine approche du vitalisme. Cependant, la juxtaposition de théories parfois improbables mais plausibles finit par former un tout cohérent qui devient convaincant. Au final, le choix d'une théorie plutôt que d'une autre a une forte composante subjective, et la théorie de Wendt ne peut pas être éliminée sur la base des connaissances actuelles. Concernant les implications pour les sciences sociales, Sven Steinmo met en avant l'intérêt du challenge posé par Wendt pour renouveler les façons de penser ${ }^{8}$. Pour Stephen DeCanio, en revanche, il n'est pas pertinent de lier les sciences sociales à des théories physiques, qui sont sujettes à des révisions constantes? Robert Jervis ajoute que la complexité qu'une approche quantique entrainerait ne nous orienterait pas mieux dans le monde social (les décideurs pouvant être submergés par la multiplication des possibles $)^{10}$. Enfin, pour Bentley B. Allan, si l'approche quantique de l'agentivité est attractive, des chercheurs en sciences sociales ont déjà travaillé depuis un certain temps sur des représentations similaires, en dehors des contraintes de la physique (en dépit du principe du "causal closure of physics»). Pour ce commentateur, "les apports d'une science sociale quantique devraient être un élément essentiel de toute théorie sociale post-classique, mais la théorisation de l'agencivité sociale ne devrait pas se faire exclusivement sous les contraintes de la physique quantique ou d'autres descriptions mathématiques ${ }^{11}$.

\footnotetext{
Daniel Little, op. cit.

Sven Steinmo, op. cit.

Stephen DeCanio, op. cit.

Robert Jervis, op. cit.

11 Bentley B. Allan, op. cit.
} 
Au final, l'ouvrage de Wendt constitue incontestablement une avancée notable dans le champ de la sociologie quantique, de façon complémentaire avec d'autres ouvrages majeurs récents sur le sujet ${ }^{12}$. Depuis sa parution, des chercheurs renommés ont commencé à mobiliser et explorer la conception quantique proposée par Wendt. C'est le cas notamment de Karen O’Brien, chercheuse de premier plan dans la communauté de recherche sur le changement climatique. Cette chercheuse utilise la théorie de la conscience quantique conjointement avec les théories de la structuration et des réseaux pour théoriser le concept de " pouvoir collaboratif ", c'est-à-dire la capacité des individus à se connecter en vue d'un objectif désiré (par exemple, limiter le réchauffement climatique à $\left.1,5^{\circ} \mathrm{C}\right)^{13}$. Dans ce sens, une idée particulièrement intéressante de Wendt, également développée par la théoricienne féministe Karen Barad ${ }^{14}$, est que les individus ne sont pas complètement séparables, mais plutôt "enchevêtrés " et " intra-agissants ", et que les structures sociales ne sont pas seulement extérieures aux individus, mais aussi « internes aux êtres humains collectivement ». Plus généralement, les approches quantiques (incluant celle de Wendt) sont aujourd'hui mobilisées dans des projets d'envergure ${ }^{15}$, et commencent à se développer dans les communautés de référence en sociologie, comme l'International Studies Association (ISA) (organisation de sessions sur les approches quantiques dans les trois derniers congrès annuels de l'association). En France, on peut mentionner par exemple les travaux pionniers d'Ariane Lambert-Mogiliansky et

12 Jerome Busemeyer et Peter Bruza, Quantum Models of Cognition and Decision, Cambridge, Cambridge University Press, 2012 ; Emmanuel Haven et Andrei Khrennikov, Quantum Social Science, Cambridge, Cambridge University Press, 2013.

13 Karen O’Brien, «Climate Change and Social Transformations: Is It Time for a Quantum Leap? ", Wiley Interdisciplinary Reviews: Climate Change, vol. 7, $\mathrm{n}^{\circ}$ 5, 2016, p. 618-626.

14 Karen Barad, Meeting the Universe Halfway: Quantum Physics and the Entanglement of Matter and Meaning, Durham (NC), Duke University Press, 2007.

15 «Projet Q» de James Der Derian, https://projectqsydney.com/. 
François Dubois ${ }^{16}$ en économie, et toute l'œuvre de Michel Bitbol $^{17}$ autour des implications de la physique quantique sur notre rapport à la réalité.

Pour conclure, il convient de rappeler le caractère très spéculatif de l'ouvrage, dont il faudra sans doute du temps pour apprécier les apports effectifs, en attente d'une vérification (ou pas) des hypothèses formulées. Dans cette perspective, certaines approches méthodologiques semblent adaptées pour mettre à l'épreuve concrètement dès aujourd'hui ces hypothèses de Wendt. En particulier, la "méthodologie $Q$ " est une technique mixte quanti-qualitative particulièrement bien adaptée à l'étude de la subjectivité, et basée sur des formalismes quantiques ${ }^{18}$. Cette méthodologie a déjà fait les preuves de sa pertinence pour rendre compte des opinions, sentiments et connaissances des individus pour une diversité de problématiques, comme la gestion de la faune sauvage en Norvège ${ }^{19}$, ou la sylviculture durable aux EtatsUnis $^{20}$. Une autre méthode adaptée est l'entretien d'introspection guidée " micro-phénoménologique », qui constitue une extension de l'entretien d'explicitation ${ }^{21}$, permettant une description précise et rigoureuse de l'expérience vécue du sujet ${ }^{22}$. En effet, le statut épistémologique de cette méthode est isomorphe à celui de la

$\overline{16}$ Ariane Lambert-Mogiliansky et François Dubois, "Our (Represented) World: A Quantum-Like Object », dans Ehtibar Dzhafarov, Scott Jordan, Ru Zhang et Victor Cervantes (dir.), Contextuality from Quantum Physics to Psychology, Hackensack (NJ), World Scientific, 2016, p. 367-386.

17 Michel Bitbol, Mécanique quantique. Une introduction philosophique, Paris, Flammarion, 1996 ; Michel Bitbol, Physique et philosophie de l'esprit, Paris, Flammarion, 2000.

18 Karen O’Brien, op. cit.

19 Yennie Bredin, Henrik Lindhjem, Jiska van Dijk, John Linnell, « Mapping Value Plurality towards Ecosystem Services in the Case of Norwegian Wildlife Management: A Q Analysis ", Ecological Economics, n ${ }^{\circ} 118,2015$, p. 198-206.

20 Paula Swedeen, " Post-Normal Science in Practice: A Q Study of the Potential for Sustainable Forestry in Washington State, USA ", Ecological Economics, vol. 57, $\mathrm{n}^{\circ}$ 2, 2006, p. 190-208.

21 Pierre Vermersch, L'entretien d'explicitation, Paris, ESF Sciences Humaines, 2019.

22 Claire Petitmengin, Michel Bitbol, Magali Ollagnier-Beldame, "Vers une science de l'expérience vécue ", Intellectica, vol. 64, n 2, 2015, p. 53-76. 
physique quantique, en ceci qu'elle ne propose pas de rechercher quelque chose qui se trouverait tout fait dans le monde, mais reconnait au contraire que les phénomènes ne sont pas indépendants de la situation expérimentale qui les rend manifestes ${ }^{23}$. Des applications de l'entretien d'introspection guidée microphénoménologique sont aujourd'hui explorées dans des domaines aussi variés que la médecine, l'enseignement et la technique ${ }^{24}$. Ces méthodes permettront peut-être de valider, infirmer ou affiner les hypothèses de Wendt et leurs implications en sciences sociales. En attendant, comme l'indique Wendt, deux niveaux de lecture de QMASS sont possibles, la théorie pouvant être prise dans un sens métaphorique, ou dans un sens littéral. Dans les deux cas, et indépendamment de sa fécondité potentielle, Quantum Mind and Social Science nous parait un ouvrage remarquable en soi par les questions de fond qu'il soulève et sa démarche résolument transdisciplinaire, associant à la fois audace et rigueur épistémologiques.

$\overline{23}$ Michel Bitbol, La conscience a-t-elle une origine? Des neurosciences à la pleine conscience. Une nouvelle approche de l'esprit, Paris, Flammarion, 2014.

24 Claire Petitmengin, Michel Bitbol, Magali Ollagnier-Beldame, op. cit. 